\title{
The role of lexical stress on vowel duration and vowel space in two varieties of Spanish
}

\author{
Fabian Santiago ${ }^{1,2,3}$, Paolo Mairano $^{4}$ \\ ${ }^{1}$ University of Paris VIII, UMR 7023, Structures Formelles du Langage, France \\ ${ }^{2}$ UMR 7018, Laboratoire de Phonétique et Phonologie (Paris 3), France \\ ${ }^{3}$ UMR 7110, Laboratoire de Linguistique Formelle (Paris 7), France \\ ${ }^{4}$ University of Turin, Italy \\ fabian.santiago-vargas@univ-paris8.fr, paolo.mairano@gmail.com
}

\begin{abstract}
This paper investigates the effects of lexical stress on vowel durations, vowel space and vowel quality in Spanish. Data come from oral productions of 22 Spanish speakers (10 from Madrid and 12 from Mexico City) performing different tasks. As for durational cues, we found that vowel durations play a role as a cue of lexical stress. Interestingly, our results also show differences between the two varieties (the stressedunstressed ratio being larger for Mexican than Madrilenian speakers). Instead, we show that the expansion/compression of the vowel space is not affected by lexical stress, but it does seem to be affected by the task type. We found, however, that lexical stress can affect vowel quality in certain cases: unstressed /a/ and /o/ tend to be centralized. We discuss these results in the light of previous research reporting effects of lexical stress on vowel spectral quality in different varieties of Spanish
\end{abstract}

Index Terms: vowel reduction, vowel space, vowel quality, lexical stress

\section{Introduction}

In lexical-stress languages, vowels in strong prosodic positions (stressed vowels) tend to be characterized by different phonetic details with respect to vowels in weak positions (unstressed vowels). Stressed vowels tend to be articulated with longer durations and higher amplitudes; they attract pitch contours, and are more dispersed within the vowel space. Conversely, unstressed vowels tend to have shorter durations and lesser amplitude, lack pitch contours, and show a tendency towards centralization (in some languages they can be fully centralized).

In the theory of [10], the distinction between stressed and unstressed vowels could be seen as a kind of hypo- and hyperarticulation (economy of the maximization of articulatory gestures). This theory has been tested in many languages (cf. [2] for English, [12] for Spanish, among others) and the findings reported in these studies show that lexical stress has an effect on the vowel formant dispersion: stressed vowels show an expansion of the vowel space whereas unstressed ones show a compression.

In Spanish, the compression of the vowel space is not seen as phonological ([8]), i.e. the five vowels /a, e, i, o, u/ are always founded in the stressed or unstressed positions. However, different studies have investigated the acoustic patterns of stressed vs unstressed vowels and found controversial results. [13] examined the phonetic cues of vowels $/ \mathrm{o} /$ and $/ \mathrm{i} /$ in stressed and unstressed positions in the production of five Barcelonan Spanish speakers. They report that unstressed vowel /o/ tend to be centralized, whereas vowel /i/ is always located in the extreme positions of the vowel chart. [14] carried out a similar experiment with 20 Castilian speakers analyzing the same vowels with the same experimental protocol and did not find any acoustic cue of vowel reduction. $[13,14]$ reported that the presence of pitch accents does not affect vowel quality in Spanish.

[12] analyzed the five Spanish vowels produced by 20 Central Iberian Spanish speakers and found that unstressed vowels /a/ and /i/ tend to be centralized, whereas unstressed /e, $\mathrm{o}, \mathrm{u} /$ remain in the similar peripheral positions as their stressed counterparts. Additionally, [5] did not find centralization of unstressed vowels in Andean Spanish, whereas [4] report that almost all unstressed vowels of Colombian Spanish (except /i/) display centralization. In all these studies, it is found that Spanish stressed vowels are produced with longer durations than unstressed vowels.

In the light of the results reported in the studies above, it appears that lexical stress has an effect on segmental durations in Spanish (see [15] for a discussion of other prosodic cues involved in the production of lexical stress, namely f0, intensity and spectral tilt). Yet, it remains unclear whether the absence of lexical stress triggers vowel centralizations, and whether dialectal differences exist in this respect between different varieties of Spanish. Inconsistencies of these results could be due to several factors. On the one hand, the type of data differs: read speech in $[12,13,14]$, spontaneous speech in [4]. On the other hand, centralizations are mainly described as formant differences between stressed and unstressed vowels (i. e., the effects observed on F1 and F2 values separately for each vowel), rather than analyzing the vowel space as a whole system $[6,12]$. Finally, it seems that vowel centralizations as a function of lexical stress could be different across Spanish varieties (cf. the results discussed above between Colombian and Andean Spanish)

This study tries to fill this gap by investigating whether the lexical stress has an effect on the $\mathrm{F} 1 * \mathrm{~F} 2$ vowel space in terms of its compression/expansion. Our goal is twofold: (i) clarify whether the vowel space (seen as a whole system) is sensitive to stress in Spanish and (ii) examine whether differences could be observed in two varieties (Madrilenian Spanish vs Mexican Spanish). We expect that the presence of lexical stress should result in an increase of vowel duration, but not necessarily a vowel space compression, given that Spanish does not have phonological vowel reduction. Additionally, we analyze the effects of stress on vowel quality for each Spanish vowel 
individually. Finally, we also investigate whether the task (read $v s$ spontaneous speech) plays a role.

\section{Data and methods}

The data for this study comes from a corpus compiled by [16]. It contains productions of 22 Spanish speakers (5.75 hours of speech, $45 \mathrm{k}$ words). $72 \mathrm{k}$ vowels were analyzed.

\subsection{Participants \& Speech Materials}

12 Mexicans (MEX) and 10 Spanish speakers (SPA) were recorded in a soundproof room (aged 21-41, $S D=5$, genderbalanced groups). Both groups were Spanish monolinguals from birth and were attending university in Mexico City and Madrid respectively at the time of recordings. Participants in the MEX group were attending their $\mathrm{BA}, \mathrm{MA}$ or $\mathrm{PhD}$ in different domains (Sciences, Arts and Sociology). SPA participants were mainly attending an MA in Applied Linguistics ( 7 out of 10 ).

All participants performed the following tasks in this order: two spontaneous speech tasks including a semi-directed interview and the description of a painting, and one read-aloud task. In the semi-directed interview, participants were asked to describe their typical days and activities at work, university, home, etc. In the second task, speakers described a colorful Renoir painting ("Dance at le Moulin de la galette"). Participants were told to describe the facial expression and clothes of the characters in the painting, the landscape and general ambiance. After this description, participants read 9 short stories and dialogues three times with a natural speech rate (not fast, not slow). Tasks 1 and 2 will be referred to as spontaneous speech, tasks 3 as read speech. For the latter, vowels were extracted from the second reading task. Each task lasted a minimum of 10 minutes approximately. Recordings were collected with a microphone and a portable wav recorder with +48 Volt phantom power at a sample rate of $44.1 \mathrm{~Hz}$ within 16-bit resolution.

\subsection{Linguistic and phonetic Annotation}

The corpus was orthographically annotated on Praat. Words presenting disfluencies, errors and all filled pauses (este, eh, $m h$ ), fragments (univer-, respon-, etc.), overlaps, noticeable lengthened and/or unrecognized words were labeled and excluded from the analysis. The corpus was segmented into words, syllables ad phones with EasyAlign [7].

Labels for segments accounted for canonical pronunciations of the words in each Spanish variety (lenitions of plosives $/ \mathrm{b}, \mathrm{d}, \mathrm{g} /$; nasal assimilations and transcriptions of glides in diphthongs). In spontaneous speech, deletions and non-canonical pronunciations are quite common, so the forced alignment included a considerable amount of errors on boundary locations. For this reason, the automatic segmentation was carefully hand-verified by the first author.

Many cases of vowel shortening and devoicing (sometimes even deletions) were found in data for Mexican Spanish. For instance, the word tesis ('thesis') could be produced as tess [3]. In cases where there was no visual evidence of vowel formants, a boundary of $10 \mathrm{~ms}$. was systematically marked between the consonants around the suppressed vowel. Finally, stressed and unstressed vowels were labeled according to the phonological rules of Spanish.

\subsection{Metrics: vowel duration and vowel space}

\subsubsection{Vowel duration}

Vowel durations were highly unbalanced due mainly to speech rate differences across participants. In order to minimize these effects, vowel durations were transformed to $z$-scores by speaker. Additionally, we excluded all vowels produced at the end of utterances and all vowels produced in prepausal positions in order to avoid the effects of final lengthening.

\subsubsection{Vowel space}

We automatically extracted different acoustical measures with a Praat script for each of the five Spanish vowels (a, e, i, o, u): durations, F1 and F2 values (extracted at the mid-point of all the vocalic segments). The amplitude peaks were detected in a band lower than $5 \mathrm{kHz}$ for males, and lower than $6 \mathrm{kHz}$ for females, with a $25 \mathrm{~ms}$ Gaussian window. Formant values were converted into Barks via an $\mathrm{R}$ script in order to make a byspeaker normalization ([9]).

Three measures of vowel space were computed from F1 and F2. The first one consisted in the 'degree of dispersion' following [6]: we identified the gravity centre of the acoustic space by averaging F1 and F2 values of all vowels produced by each speaker; (ii) we computed the Euclidean distances from the gravity centre to each vowel's position within the F1F2 chart, then averaged them for the 5 peripheral Spanish vowels (separately for stressed and unstressed vowels); (iii) we summed the resulting averaged Euclidean distances for the 5 peripheral Spanish vowels (separately for stressed and unstressed vowels and type of task).

The second and third metrics consisted in the area of the polygon defined by the F1*F2 means for each Spanish vowel and the area of the convex hull encompassing all measured vowel tokens [1, 11]. Similarly to Euclidean distances, the polygon area and convex hull were obtained separately for each speaker under the conditions of stressed and unstressed vowels and type of task. The three metrics of vowel space are illustrated in Figure 1.

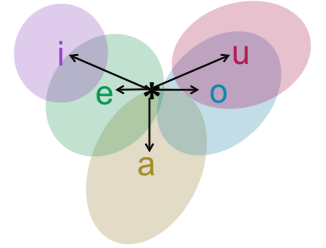

(A)

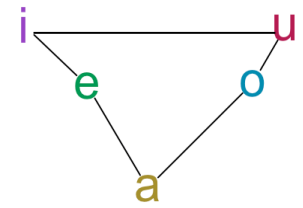

(B)

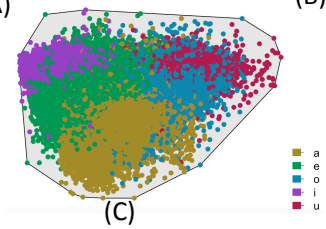

Figure 1: Illustrations of Euclidean distances (A) [6],

Polygon Area (B) [1] and Convex Hull Area (C) [11].

We applied a filter to exclude potential errors in formant detection and aberrant formants values associated to phonemes not physically present but labeled in the corpus. Finally, vowels in diphthongs emerging as glides, i.e., [i] in the word peine ('comb') or [u] in the word aula ('classroom') were discarded from the analysis. The remainder of the analysis was 
carried out on 56,733 vowels. Table 1 shows counts for the vowels analyzed.

Table 1: Number of vowels by variety.

\begin{tabular}{ccc}
\hline & SPA & MEX \\
\hline a & 7,336 & 9219 \\
e & 8934 & 11427 \\
$\mathrm{i}$ & 2447 & 2854 \\
$\mathrm{o}$ & 5110 & 6453 \\
$\mathrm{u}$ & 1190 & 1763 \\
\hline
\end{tabular}

\section{Results}

The statistical analysis was carried out using linear mixedeffect models. Different models were constructed for each dependent variable (vowel duration and vowel space obtained with three metrics). We considered the following independent variables: Language (SPA vs MEX), Stress (stressed vs unstressed), Task (read $v s$ spontaneous), and Vowel (a, e, i, o, u). Random intercepts for participants were analyzed and, depending on the analysis, a random style slope for participants was included as well. We assessed the contribution of fixed factors and interactions with likelihoodratio tests between full and reduced models.

\subsection{Effects of lexical stress on vowel durations}

The first question in this study was to evaluate whether lexical stress affects vowel durations in the two varieties of Spanish. As expected, the statistical analysis evaluating the effects of three-way interactions between stress, language and task on vowel durations showed that stressed vowels are longer that unstressed vowels $(\beta=0.126, S E=0.026, t=4.75, p<$ $.0001)$. We found that language is also a predictor of vowel durations: vowels, stressed or not, tend be shorter in MEX than in SPA $(\beta=-0.043, S E=0.015, t=-2.87, p<.001)$. More interestingly, the interaction 'stress $\mathrm{x}$ language' was also significant indicating that the difference of durations between stressed $v s$ unstressed vowels is larger in MEX than for SPA $(\beta=0.128, S E=0.036, t=3.57, p<.001)$. Finally, clear differences between the tasks were found: vowel durations in the reading task decrease compared to the spontaneous speech $(\beta=-0.152, S E=0.041, t=3.65, p<.001)$. The effects of interactions between 'stress $\mathrm{x}$ task' and 'stress $\mathrm{x}$ task $\mathrm{x}$ language' were not statistically significant (all $p$-values $>.05$ ).

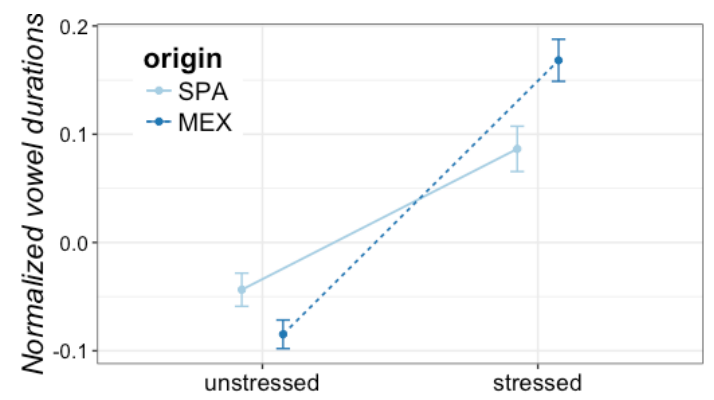

Figure 2: Vowel durations according to stress status and language.

We thus conclude that vowel durations are affected by the presence of stress in both languages as expected, but not in the same way. Figure 2 above illustrates the interaction between 'stress x language': speakers produce stressed vowels with longer durations than the unstressed ones but the magnitude of the difference is bigger in MEX than in SPA. Spanish speakers reduce unstressed vowels by $7 \%$ with respect to their stressed counterparts, whereas Mexican speakers reduce them by $13 \%$.

\subsection{Effects of lexical stress on the vowel space}

The second question in this study was to clarify whether the lexical stress has an effect on the vowel space in these languages. The analysis in section 3.1 shows that stress is a good predictor of vowel duration, as has been largely pointed out in the literature $[8,13,15]$. The theory of $[10]$ argues that languages marking the contrasts between stressed $v s$ unstressed vowels with durational cues tend to modify the vowel quality as well, i.e., there is a correlation between short durations and vowel space compression. Since the contrast between stressed $v s$ unstressed syllables is more important in MEX than in SPA, we investigate whether MEX would show a greater deal of vowel compression than SPA (or some $v s$ no compression).

Table 4 summarizes the average vowel space with the three metrics used in this study according to lexical stress and language. In order to validate statistically these differences, we run three different models with the sum of Euclidean distances, Polygon Area and Convex Hull Area as dependent variables with stress, language and task as fixed factors (and all their possible interactions).

Table 4: Vowel space metrics (Barks ${ }^{\wedge}$ ) by stress \& language

\begin{tabular}{lcccccc}
\hline & \multicolumn{2}{c}{ Euc. Distances } & \multicolumn{2}{c}{ Polygon Area } & \multicolumn{2}{c}{ Convex Hull Area } \\
\hline & SPA & MEX & SPA & MEX & SPA & MEX \\
Stress & 31.95 & 28.18 & 6.72 & 5.52 & 41.31 & 38.21 \\
Unst. & 31.49 & 27.12 & 6.43 & 5.14 & 43.16 & 39.58 \\
\hline
\end{tabular}

The results confirmed that speakers in both languages do not compress the vowel space when producing unstressed vowels: the effects of stress and language on vowel space did not reach statistical significance for any of the three metrics (all $p$-values $>.05$ ). Interestingly, despite the fact that MEX speakers tend to mark the contrast between stressed vs unstressed vowels with greater durational differences than SPA, the vowel space in MEX does not seem to be sensitive to the presence/absence of lexical stress. The analysis also showed that the interactions 'stress $x$ language' on the three metrics accounting for the vowel space were not statistically significant: all the $p$-values $>.05$ suggest that the absence of lexical stress does not trigger any kind of vowel space compression. The output of these models indicates that the MEX vowel space tends to be smaller than SPA in terms of degree of dispersion and polygon area $(p=.09$ and $p=.08$ respectively). These marginal differences, however, are only an indicator of the different properties of these two vowel systems without any correlation with stress.

Instead, we found an effect of vowel space for task type: speakers show a tendency to increase the vowel space in spontaneous speech vs read speech, in terms of Euclidean Distances and Convex Hull Area $\left(\beta_{\text {Euc. D }}=3.21, S E=1.27, t=\right.$ $\left.2.52, p<.01 ; \beta_{\text {Con. Hull }}=9.74, S E=1.85, t=5.25, p<.0001\right)$. As for the Polygon Area, we found an interaction between 'stress $\mathrm{x}$ task' suggesting that in read speech, speakers tend to increase the vowel space of unstressed vowels with respect to the spontaneous speech, whereas in the case of stressed 
vowels, there are no effects $(\beta=-1.05, S E=0.37, t=-2.79, p$ $<.001)$.

These results clarify that differences reported in Table 4 are not statistically significant, suggesting that in both varieties, lexical stress does not trigger any kind of vowel space compression. Instead, task seems to be a good predictor of vowel space compression, and in both Spanish varieties. [4] found that in read speech, Colombian Spanish vowels are more peripheral in the vowel chart than vowels in spontaneous speech. Conversely, in our data of Madrilenian and Mexican Spanish almost all vowels tend to be centralized in read speech $v s$ spontaneous speech, as shown in Figure 3.

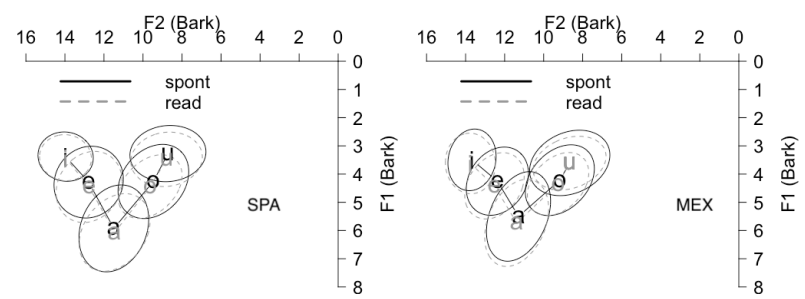

Figure 3: Vowel plots of spontaneous vs read speech \& variety

\subsection{Effects of lexical stress on the vowel quality}

The final question was to analyze the effects of lexical stress on vowel quality for each Spanish vowel individually. Figure 4 illustrates vowel plots for the five Spanish vowels produced by SPA (left) and MEX (right) groups according to their lexical status. Looking at the mean values of each vowel, one can notice than stressed vowels tend to be more peripheral on the vowel chart than their unstressed counterparts in both languages.

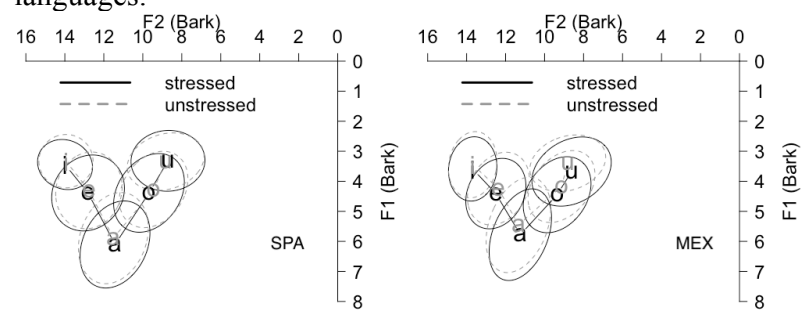

Figure 4: Vowel plots of stressed vs unstressed vowels \& variety

This plot illustrates that not all vowels modify their vowel quality in the same way, as $[4,12,13]$ have pointed out previously. Nonetheless, overlaps do not allow to see this clearly. In order to see whether lexical stress affects vowel quality, we obtained the delta of Euclidean distances from the gravity centre to the mean of stressed vowels and the Euclidean distance from the centroid to their unstressed counterparts by speaker. We ran five different mixed-effect models for each vowel individually estimating the effects of language and stress on delta values. The results showed that there are main effects of stress on vowel quality for vowels /a/ and $/ \mathrm{o} /$. We found that Euclidean distances for vowel /a/ from the centroid in unstressed vowels decrease from 3.38 to 2.86 Barks $^{\wedge} 2(\beta=.44, \mathrm{SE}=.11, \mathrm{t}=3.761, \mathrm{p}<.01)$ indicating that speakers articulate this unstressed vowel with less aperture. As for vowel $/ \mathrm{o} /$, the same pattern is observed: unstressed /o/ vowels are farther from the centroid than their stressed counterparts and Euclidean distances increase from 6.77 to 7.65 Barks ${ }^{\wedge} 2$ indicating an assimilation to $/ \mathrm{u} /$ vowel $(\beta=-.98$, $\mathrm{SE}=.22, \mathrm{t}=-4.45, \mathrm{p}<.0001)$. Vowels $/ \mathrm{i}, \mathrm{e}, \mathrm{u} /$ are not sensitive to stress (all $p$-values $>.05$ ) in both languages. The language and the interaction 'stress $\mathrm{x}$ language' were not significant either (all $p$-values $>.05$ ).

\section{Discussion \& Conclusion}

The findings reported in this study suggest that lexical stress; tasks and language have main effects on vowel durations of Madrilenian and Mexican Spanish speakers. Stressed vowels are longer than their unstressed counterparts as it has been claimed by $[8,12,13,15]$. Moreover, the temporal phonetic cues observed in both languages differ in the two varieties: Mexican speakers make the stress contrast with greater durational differences than Spaniards. The task affects the vowel duration as well. The longer durations observed in spontaneous speech probably reflect differences of articulation rate. As it has been pointed out by [16], the spontaneous speech tasks demand more complex cognitive functions than read speech: slower articulation rates in such tasks presumably reflect higher cognitive load.

Regarding the metrics of vowel space, our results support findings by [4] that the task has a main effect on vowel space compression. However, our results go in the opposite direction, with a more compressed vowel space for read speech, whereas [4] found that Colombian speakers rather tend to expand vowel space in read speech. One could logically expect that speakers articulate with a careful pronunciation in read-aloud tasks resulting in the expansion of the vowel space. However, a possible explanation for our findings is that spontaneous speech is related to slow speech rates, whereas read speech is characterized by fast speech rates. Consequently, the vowel space expansion/reduction could rather be a result of durational differences triggered by different speech rates.

Finally, the dispersion of vowel space measured with 3 types of metrics clearly shows that there is no vowel space compression when producing unstressed vowels in both languages. However, these observations do not imply that specific vowels can be affected by stress: in fact, unstressed /a/ and $/ \mathrm{o} /$ did show some degree of centralization.

Our results suggest that centralization phenomena in languages without phonological reduction like Spanish may need to be interpreted differently. We propose that different vowel qualities do not necessarily imply vowel space compression.

A limitation of this study is that we do not analyze the effects of prominence levels (the production of pitch accents) on vowel durations and vowel space. This type of analysis was not carried out due to the huge effort needed to annotate pitch accents for a large amount of data. It is not impossible then that the effect of pitch accents could affect the vowel space. Yet, $[13,14]$ pointed out that the presence of pitch accents is not a good predictor for vowel space expansion/compression. Although this point may be further explored in future research, we find it reasonable to assume that pitch accents do not heavily affect the vowel space expansion/compression in Spanish. 


\section{References}

[1] A. R. Bradlow, G. M. Torretta and D. B. Pisoni, "Intelligibility of normal speech I: Global and fine-grained acoustic-phonetic talker characteristics," Speech Communication, no. 20, pp. 255272, 1996.

[2] T. Cho, "Prosodic strengthening and featural enhacement: Evidence from acoustic and articulatory realizations of $/ \mathrm{a}, \mathrm{i} / \mathrm{in}$ English,". Journal of the Acoustical Society of America, no. 117, pp. 3867-3878.

[3] M. J. Canellada, A. Zamora, "Vocales caducas en el español mexicano," Nueva Revista de Filología Hispánica, no. 14, pp. 222-241, 1960.

[4] J.A. Correa Duarte, "Caracterización acústica de la reducción vocálica en el español de Bogotá (Colombia)," Estudios de Fonética Experimental, no. 26, pp. 63-91, 2017.

[5] A. M. Delforge, "Unstressed Vowel Reduction in Andean Spanish," Selected Proceedings of the $3^{\text {rd }}$ Conference on Laboratory Approaches to Spanish Phonology, L. Colantoni \& J. Steele (eds), Somerville, MA: Cascadilla Proceedings Project, pp. 107-124, 2008.

[6] C. Gendrot and M. Adda-Decker, "Impact of duration and vowel inventory size on formant values of oral vowels: an automated formant analysis from eight languages," Proceedings of the $16^{\text {th }}$ International Conference of Phonetic Sciences, pp. 1417-1420, 2007.

[7] J.-P. Goldman, "EasyAlign: An automatic phonetic alignment tool under Praat," Proceedings of Interspeech, pp. 3233-3236, 2011 .

[8] J. I. Hualde, The sounds of Spanish. Cambridge, UK: Cambridge University Press, 2005.

[9] W. Labov, "A sociolinguistic perspective on sociophonetic research," Journal of Phonetics, no. 34, 500-515, 2006.

[10] B. Lindblom. "Explaining phonetic variation: A scketch of the H\&H theory," in Hardcastle \& Marchal (eds), Speech Production and Speech Modeling, Dorcrecht: Kluwer, pp. 403439, 1990

[11] D.R. McCloy, "Prosody, intelligibility and familiarity in speech perception," Doctoral Dissertation, University of Washington, 2013

[12] M. Nadeu, "Stress- and speech rate-induced vowel quality variation in Catalan and Spanish," Journal of Phonetics, no. 46, pp. 1-22, 2014.

[13] M. Ortega-Llebaria and P. Prieto. "Disentangling stress from accent in Spanish: Production patterns of the stress contrast in deaccented syllables," in P. Prieto, J. Mascaró and M.J. Solé (eds), Segmental and Prosodic Issues in Romance Phonology, Amsterdam, Netherlands, Philadelphia PA: John Benjamins, pp. 155-175, 2007.

[14] M. Ortega-Llebaria and P. Prieto. "Acoustic correlates of stress in Central Catalan and Castilian Spanish," Language and Speech, no. 54, pp. 73-97, 2011.

[15] A. Quilis, Tratado de fonética y fonología españolas. Madrid: Gredos.

[16] F. Santiago \& P. Mairano. "Do Spaniards speak faster than Mexicans? Studying Spanish Rhythm in Natural Speech,' Submitted. 